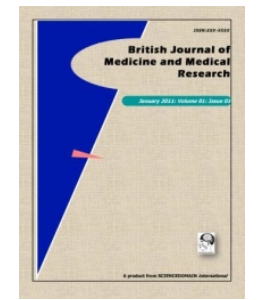

British Journal of Medicine \& Medical Research 2(3): 469-483, 2012

SCIENCEDOMAIN international

www.sciencedomain.org

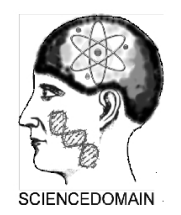

\title{
Metabolite Space of Rheumatoid Arthritis
}

\author{
Herman van Wietmarschen ${ }^{1,2^{*}}$ and Jan van der Greef ${ }^{1,2,3}$ \\ ${ }^{1}$ Division of Analytical Biosciences, LACDR, Leiden University, Leiden, The Netherlands. \\ ${ }^{2}$ Sino-Dutch centre for Preventive and Personalized Medicine, Zeist, The Netherlands. \\ ${ }^{3}$ TNO, P.O. Box 360, 3700 AJ, Zeist, The Netherlands.
}

\section{Authors' contributions}

This work was carried out in collaboration between all authors. HvW conceived the concept and ideas for this manuscript, and wrote the manuscript. JvdG contributed ideas for the manuscript and commented on the text. All authors read and approved the final manuscript.

Review Article

Received $24^{\text {th }}$ May 2012

Accepted $7^{\text {th }}$ July 2012

Online Ready $11^{\text {th }}$ July 2012

\section{ABSTRACT}

Metabolites play numerous roles in the healthy and diseased body, ranging from regulating physiological processes to providing building blocks for the body. Therefore, understanding the role of metabolites is important in elucidating the etiology and pathology of diseases and finding targets for new treatment options. Rheumatoid arthritis is a complex chronic disease for which new disease management strategies are needed. The aim of this review is to bring together and integrate information about the various roles that metabolites have in rheumatoid arthritis.

An extensive PubMed search is conducted to collect the relevant manuscripts. The metabolites are discussed in relation to rheumatoid arthritis. Subsequently, the metabolites are organized according to levels of system organization. In the last section an integrated pathway analysis of the metabolites conducted with Ingenuity Pathway Analysis software is presented.

Literature search resulted in information about vitamins, eicosanoids, fatty acids, lipids, hormones and peptides. The metabolites could be related to metabolic processes, oxidative stress processes and inflammatory processes. Cell death, lipid metabolism and small molecule biochemistry were found by the pathway analysis to be the top functions, characterized by the metabolites arachidonic acid, ascorbic acid, beta-carotene, cholecalciferol, hydrocortisone, keratan sulfate, melatonin, palmitic acid and stearic acid. These nine metabolites are highly connected to a number of canonical pathways related to immune functions, the production of nitric oxygen and reactive oxygen species in 
macrophages and pathways involved in arthritis.

This review indicates groups of metabolites that could be interesting for metabolomics studies related to rheumatoid arthritis. Circadian rhythms of metabolite levels are found to be important for understanding and treating rheumatoid arthritis. In addition, some key processes and pathways are found by integrating the metabolite data. This might offer new ideas for studies into the mechanism of and possible treatment options for rheumatoid arthritis.

Keywords: Rheumatoid arthritis; metabolites; systems biology; health space; diagnosis.

\section{INTRODUCTION}

Metabolites, the intermediates and products of metabolism, play numerous roles in the healthy and diseased body, ranging from regulating physiological processes to providing building blocks for the body. Together with genomics, transcriptomics and proteomics, metabolomics provides the biochemical building blocks for understanding the body as a complex and dynamic system of interactions (Wishart et al., 2007). Metabolites occupy a special place in this systems biology approach in that they are usually close to the phenotype (vd Greef et al., 2007). Therefore understanding the role of metabolites is very important in elucidating the etiology and pathology of diseases and finding targets for new treatment options. Metabolic profiles of cells, tissues or body fluids can provide snap-shot information about the current condition of (part of) the system. However, the future will be more determined by dynamic metabolic profiling. Measuring changes in metabolism over time and in response to challenges to the system provides a more profound insight not only in the status of the living system but also in its capacity to regulate and adapt.

Especially for chronic diseases such as Rheumatoid Arthritis (RA) of which the etiology is unclear but with both genetic and environmental factors playing a role, it is interesting to study the metabolome (Glocker et al., 2006). RA is a chronic systemic autoimmune disease characterized by inflammatory polyarthritis which affects approximately $0.5-1 \%$ of the population worldwide (Hochberg et al., 2007). Although the currently favored treatment regime using disease-modifying anti-rheumatic drugs (DMARD's) in an early stage of RA is believed to have a favorable result on disease course by influencing inflammation, patients assessment of disease activity and functional disability do not always support this result (Welsing et al., 2005). Even $30 \%$ of the RA patients initiating the most effective treatment option available, anti-TNF $\alpha$ therapy, fail to respond (Smolen, 2005). New disease management strategies are therefore needed.

In this review the focus is on metabolites involved in RA. Various hypotheses about the role of certain metabolites in the etiology and progression of RA are reviewed. Secondly, an attempt is made to elucidate relevant interactions between the metabolites identified in the RA literature making use of pathway analysis tools. Finally we show the relevance of metabolites in the RA field and provide ideas for new insights on tackling this disabling disease. 


\section{METABOLITES AND METABOLIC PROCESSES INVOLVED IN RA}

An extensive literature search of the PubMed database was performed using several key words related to metabolites in combination with the keyword 'rheumatoid arthritis'. The results were screened and categorized, leading to a number of topics with sufficient material to be discussed for this review. In this section research is discussed concerning vitamins, eicosanoids, fatty acids, lipids, hormones and cartilage breakdown products. Although trace elements are not considered metabolites, information about this particular group of micronutrients is included because of the importance for RA.

\subsection{Vitamins}

Vitamin $D$ deficiency is related to several autoimmune diseases including type 1 diabetes, multiple sclerosis, SLE and rheumatoid arthritis (Arnson et al., 2007). Vitamin D (a secosteroid) is transformed in the liver and kidney into the hormone 1,25 dihydroxyvitamin $D$ $(1,25(\mathrm{OH}) 2 \mathrm{D} 3)$, the active form that stimulates the intestinal absorption of calcium and phosphor. Vit $D$ receptor is expressed on mononuclear cells, dendritic cells, activated $T$ and $B$ cells, antigen presenting cells. Dendritic cells produce vit $D$ hormone which inhibits T-cell proliferation, maturation of dendritic cells, secretion of IL-2 and INF-y by CD4+ T-cells, secretion of cytokines by macrophages and B-cell antibody production (Arnson et al., 2007). Low blood levels of vit $D$ hormone also result in increased blood levels of insulin-like growth factor (IGF-1), a potent stimulator of cell growth and inhibitor of apoptosis (Huynh et al., 1998, O'Connor et al., 2008). In short, Vit D hormone stimulates an anti-inflammatory environment and particularly affects Th1 driven autoimmunity (Cutolo et al., 2007).

There is some controversy concerning the association of vit $D$ intake with RA risk. However, Cutolo et al. (2006) suggest that these differences in results arise from the various ways of measuring vitamin $D$ intake. Vitamine $D$ intake should be measured in serum and not by dietary questionnaires (Cutolo et al., 2011). When this is taken into account, low levels of vitamin $\mathrm{D}$ can be associated with an increased disease risk as well as increased disease activity (Merlino et al., 2004; Oelzner et al., 1998). Several factors can result in lower blood vit $D$ hormone levels. Animal protein has been shown to block the kidney enzyme responsible for converting vit $D$ to the active vit $D$ hormone by promoting blood acidity (Breslau et al., 1988; Langman, 1989). Secondly, high blood calcium levels decreases vit D hormone which regulates blood calcium levels (Chan et al., 1998).

Interestingly a circannual rhythm in Vit $D$ levels was found which correlates inversely with DAS28 scores of RA patients, which could also provide difficulties in interpreting measurements (Cutolo et al., 2006). Taking this into account lower serum levels of vit $D$ seem to be associated with higher disease activity. This suggests that vit $D$ might play a role in the differences in RA prevalence between northern and southern countries (Cutolo et al., 2006).

Other vitamins with antioxidant activity such as vitamin $C$, vitamin $E$ and $\beta$-carotene have also been implicated in RA. Pattison et al. (2005) found a threefold increased risk of developing inflammatory polyarthritis when vitamin $\mathrm{C}$ intake was very low (Pattison et al., 2005). Low serums levels of $\beta$-carotene have been detected in blood bank samples of people who later on developed RA (Comstock et al., 1997). Low levels of plasma pyridoxal5-phosphate (PLP) the active form of vitamine B6 have been found in RA patients (Roubenoff et al., 1995). In general, a low antioxidant status increases the risk of developing 
RA (Heliovaara et al., 1994). No relationships have been found between the progression of $R A$ and plasma vitamin $D$, vitamin $C$, vitamin $E$ and $\beta$-carotene levels.

\subsection{Eicosanoids and Fatty Acids}

RA patients have been shown to improve on a diet low in arachidonic acid (AA) and supplemented with N-3 fatty acids (Adam et al., 2003). Arachidonic acid and other fatty acids are precursors for eicosanoids which are important mediators of inflammation. Interestingly $\mathrm{N}-6$ and $\mathrm{N}-3$ fatty acids are competitively metabolized into N-6 and N-3 eicosanoid families with different functions. An N-6 dominant diet is implicated in increased prevalence of thrombotic vascular events and inflammatory diseases. The modern diet contains over 50 times the required amount of $\mathrm{N}-6$ fatty acids which markedly shifts the $n-6 / n-3$ ratio (Cleland and James, 1997). N-3 fatty acids are less pro-inflammatory and have been shown to reduce TNF- $\alpha$ and IL- $1 \beta$ levels in RA patients, by competitively inhibiting the production of leucotriene B4 (LTB4) from arachidonic acid (Kremer et al., 1990).

Arachidonic acid is metabolised in several classes of compounds denoted as eicosanoids including prostaglandins, thromboxanes, leukotrienes, hydroxy- and epoxy-fatty acids, lipoxins and isoprostanes (Funk, 2001). Leukotriene B4 and 5-hydroxy-eicosatetraenoic acid (5-HETE) are two strong chemotactic eicosanoids found in synovial fluid of RA patients (Klickstein et al., 1980). Dietary AA is exclusively derived from animal based foods of which $80 \%$ is metabolized into eicosanoids. Endogenous AA synthesis from linoleic acid also occurs in the body, which is inhibited by poly-unsaturated fatty acid (Adam et al., 2003). These prostaglandin synthesis pathways are the basis of NSAID's and are therefore essential in current RA management strategies (ACR, 2002).

\subsection{Lipids}

An atherogenic blood lipid profile has been reported in persons who more than 10 years later developed RA, characterized by higher total cholesterol, triglyceride and apolipoprotein B levels and lower high-density lipoprotein (HDL) cholesterol levels (van Halm et al., 2007). Contradictory observations have been reported concerning changed lipid profiles in RA. Dursunogh reported higher levels of $L p$ (a) lipoprotein, triglyceride (TG), lower levels of HDL cholesterol and no changes in total cholesterol and low-density lipoprotein (LDL) cholesterol (Drusunoglu et al., 2005). Lazarevic also reported increased TG and decreased HDL-C levels, but found lower total cholesterol (TC) and LDL-c levels (Lazarevic et al., 1993). Lakatos in contrary reported lower TG and higher TC and LDL-c levels, but also reported lower HDL-c levels (Lakatos and Hárságyi, 1988). Only higher lipoprotein $L p(a)$ and lower HDL-c levels are consistently reported.

Ethnic, racial and geographic differences are suggested as possible causes for these differences in lipid profiles. Both genetic and dietary factors may contribute to higher $\operatorname{Lp}(a)$ levels, especially increased fat and cholesterol consumption (Cesur et al., 2007). Blood cholesterol levels increase most effectively by consuming animal protein and to a lesser extent by consuming saturated fat and cholesterol. In contrast, plant based foods contain no cholesterol and decrease blood cholesterol levels (Kritchevsky et al., 1982).

A high LDL/HDL ratio, which indicates a less favorable lipid profile, has been associated with the pro-inflammatory molecules CRP, IL-6 and TNF- $\alpha$ (Hulthe and Fagerberg 2002). In contrast HDL cholesterol associated with apolipoprotein A-1 has anti-inflammatory effects. 
Apo A-1 is reported to inhibit IL-1, TNF- $\alpha$ and interactions between T-cells and monocytes (Burger and Dayer, 2002; Hyka et al., 2001). This suggests that a less favorable lipid profile stimulates a pro-inflammatory status which might be related to RA disease activity and RA susceptibility.

\subsection{Hormones}

Cortisol is the strongest endogenous anti-inflammatory substance in the human body. Inflammation activates the immune system and the hypothalamic-pituitary-adrenal (HPA) axis, leading to the secretion of cortisol. However, in RA patients cortisol levels are not sufficiently increasing in response to inflammation (Neeck et al., 1990). Even in the presence of high serum levels of circulating cytokines, plasma cortisol levels in RA patients are similar to that of control subjects. A result of the relatively low plasma cortisol levels and presence of vasodilatory cytokines such as TNF- $\alpha$ is an increase in sympathetic activity. This is needed to stabilize blood pressure, systemic circulation and glucose homeostasis. However, the higher sympathetic tonus does not lead to an increase of noradrenaline, which has an anti-inflammatory effect, and therefore doesn't compensate the relatively low cortisol levels (Capellino and Straub, 2008).

Inflammatory cytokines stimulate the conversion of anti-inflammatory adrenal androgens into estrogens. Elevated estrogen and estrone levels in the synovium of RA patients sustain a pro-inflammatory state. This is thought to be related to the increased prevalence of RA in women (Capellino and Straub 2008).

It is well known that RA symptoms such as stiffness and pain can differ greatly during the day and even during the year (Labreque et al., 1995). Cutolo extensively studied metabolic processes underlying such circadian, circamensual and circannual rhythms in RA (Cutolo et al., 2005). Cortisol, melatonin and prolactin show a marked daily rhythm. Melatonin stimulates Th1 type cytokines while cortisol stimulates Th2 type cytokines with a diurnal rhythmicity (Cutolo, 2008). In patients with a high disease activity cortisol is downregulated during the evening and night, leading to an increase of inflammation in the morning. Low cortisol levels reduces its IL-6 and TNF inhibitory effects and consequently drives an increase in TNF, IFN- $\gamma$, IL-2, IL-12 and IL-6 levels. In RA patients, a melatonin plateau of 2-3 hours is observed and prolactin levels are significantly higher during the night. As a result, a Th1 response is induced during the night (Cutolo and Straub, 2008). Rhythms in concentrations of inflammatory metabolites have to be considered when time-points for measurements and treatment are being determined.

\subsection{Cartilage Breakdown Products}

RA is characterized by extensive degradation and synthesis of joint cartilage which consists for a large part of type II collagen fibers, other types of collagen and proteoglycans. Consequently, cartilage breakdown products of type II collagen have been extensively studied in the synovium, serum and urine of RA patients (Charni et al., 2005). An immunoassay is described that detects C-terminal crosslinking telopeptide of type II collagen (CTX-II) (Christgau et al., 2001). Elevated urine CTX-II levels have been associated with more rapid disease progression in OA and RA patients. Another urine marker for early RA and progression of RA is HELIX-II, a sequence arising from degradation of the helical region of type II collagen (Charni et al., 2005). 
Another collagen breakdown product that has been used as a biomarker in synovial fluid and serum is cross linked carboxyterminal telopeptide of type I collagen (ICTP). Markers for collagen synthesis that have been used are the aminoterminal propeptides of type I procollagen (PINP) and of type III collagen (PIIINP) (Aman et al., 1999). One study reported an increase of serum PIIINP and ICTP levels in 50\% of the RA patients. Additionally PINP and PIIINP were both increased in synovial fluid indicating collagen synthesis (Hakala et al., 1995). A disturbed balance of collagen breakdown and synthesis seems to be evident in inflamed joints.

Apart from various types of collagen, cartilage also contains large proteoglycans including the glycosaminoglycans keratan sulfate (KS) and chondroitin sulfate (CS). KS and CS containing fragments have been measured in synovial fluid as biomarkers of cartilage metabolism. In RA patients, the level of 846 fragment of CS is reported to be increased in serum and more so in synovial fluid, indicating aggrecan synthesis in the inflamed joint. Serum KS levels were reduced in RA (Poole, 1994). It is important to note that metabolite concentrations and changes can differ markedly between synovium and serum, providing multiple sources of information concerning local and systemic aspects of the disease.

\subsection{Trace Elements}

Zinc and copper are two trace elements of which plasma concentrations decrease during acute inflammation (Halsted and Smith, 1970; Lewis, 1984). Plasma zinc levels are found to be decreased in RA patients. Especially T-cell function is impaired by reduced zinc availability leading to reduced T-cell numbers and the Th1/Th2 ratio shifting towards Th2 (Honscheid et al., 2009). Zinc and copper have antioxidant properties. They are constituents in cytoplasmic superoxide dismutase (SOD), a metalloenzyme with anti-inflammatory properties, and copper is also found in the serum antioxidant ceruloplasmin (Honkanen et al., 1991). Intracellular SOD activity has been shown to be decreased in RA patients (Rister et al., 1978) which corresponds with decreased plasma zinc levels. However, the ceruloplasmin levels in serum of RA patients are increased. An increase in zinc consumption is correlated with a decrease in copper absorption by the intestine (Honkanen et al., 1991). This decrease of copper absorption might be a reason for the contradictory results found in studies into the effects of dietary zinc supplementation in RA (Rasker and Kardaun, 1982; Mattingly and Mowat, 1982; Tudor et al., 2005).

Selenium (Se) is another trace element that plays a role in regulating ROS through incorporation into selenoproteins. Glutathion peroxidase for example catalyzes the degradation of peroxides, is involved in arachidonic acid metabolism and can affect leukotrine and prostaglandine synthesis (Kalpakcioglu and Senel, 2008). Low levels of Se have been reported in plasma and cells of RA patients (Tarp et al., 1985). Selenoproteins can have various effects on the immune system such as stimulating a Th1-type response more than a Th2-type response. However Se supplements only seem to boost the immune system for people who already had low Se levels (Hoffmann and Berry, 2008). Much still needs to be learned about the functions of the various selenoproteins. 
Table 1. Organization level of metabolite changes in rheumatoid arthritis

\begin{tabular}{|c|c|c|c|}
\hline & Metabolite* & Matrix $^{* \star}$ & Effect \\
\hline Inflammation & $\begin{array}{l}\text { Vitamin D } \downarrow \\
\text { AA } \uparrow \\
\text { LTB-4 } \uparrow \\
5-H E T E \uparrow \\
\text { LDL } \uparrow \\
\text { HDL-c } \downarrow \\
\text { Lp(a) } \uparrow \\
\text { Cortisol } \downarrow \\
\text { Estrogen } \uparrow \\
\text { Prolactin } \uparrow \\
\text { Melatonin } \\
\text { n-3 FA } \downarrow \\
\text { n-6 FA } \uparrow \\
\text { VLDL } \uparrow\end{array}$ & $\begin{array}{l}p \\
p \\
s \\
s \\
p, s \\
p, s \\
p \\
p \\
p \\
p \\
p \\
p \\
p \\
s\end{array}$ & 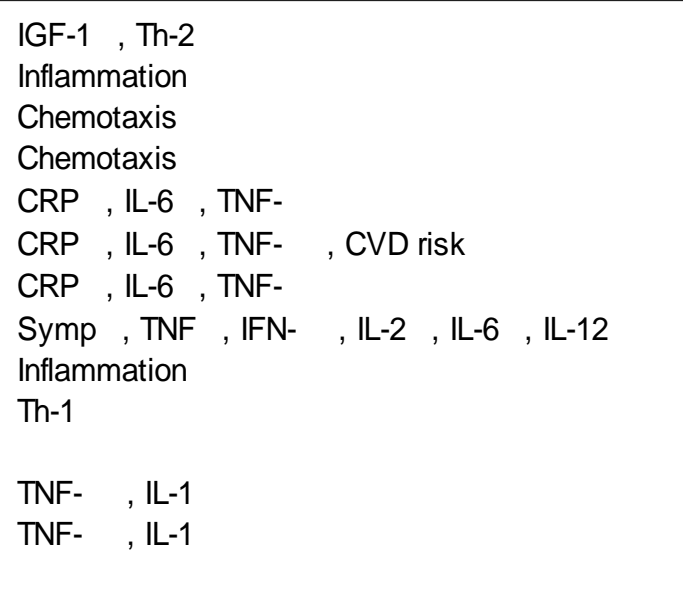 \\
\hline Oxidative stress & $\begin{array}{l}\text { Vitamin B6 } \downarrow \\
\text { Vitamin } C \downarrow \\
\text { Vitamin } E \downarrow \\
\beta \text {-Carotene } \downarrow \\
\text { Copper } \downarrow \\
\text { Znc } \downarrow \\
\text { Selenium } \downarrow \\
\text { Lactic acid } \uparrow \\
\text { Glucose } \downarrow\end{array}$ & $\begin{array}{l}p \\
p \\
p \\
p \\
p \\
p \\
p \\
p, s \\
s\end{array}$ & $\begin{array}{l}\text { RA risk } \uparrow \\
\text { RA risk } \uparrow \\
\text { RA risk } \uparrow \\
\text { RA risk } \uparrow \\
\text { SOD } \downarrow \\
\text { SOD } \downarrow \\
\text { ROS } \uparrow, \text { Th- } 2 \uparrow \\
\text { p: oxidative damage } \uparrow, \text { s: anaerobic metabolism } \uparrow \\
\text { Anaerobic metabolism } \uparrow\end{array}$ \\
\hline Metabolism & $\begin{array}{l}\text { CTXI } \uparrow \\
\text { HELIXI } \uparrow \\
\text { ICTP } \uparrow \\
\text { PIIINP } \uparrow \\
\text { KS } \uparrow \\
\text { CS } \uparrow\end{array}$ & $\begin{array}{l}u \\
u \\
p, s \\
p, s \\
p \\
p, s\end{array}$ & $\begin{array}{l}\text { RA progression } \uparrow \\
\text { RA progression } \uparrow \text {, collagen breakdown } \uparrow \\
\text { Collagen breakdown } \uparrow \\
\text { Collagen synthesis } \uparrow \\
\text { Cartilage synthesis } \uparrow\end{array}$ \\
\hline
\end{tabular}




\subsection{Metabolites and Levels of System Organization}

Van Ommen and others described a health space based on metabolomics phenotypes to organize metabolite data (van Ommen et al., 2008). The metabolites reviewed in the previous sections can be organized according to this approach in what might be called levels of system organization. In this review a distinction could be made between metabolites involved in metabolic processes, especially those located in the inflamed joints, oxidative stress processes and inflammatory processes (Table 1). As could be expected a number of compounds are upregulated or downregulated in RA patients that are involved in the inflammatory process. In addition, oxidative stress factors also seem to play an important role in RA. The metabolism aspects are mostly represented by the changes in the inflamed joints of RA patients.

\section{RA AND METABOLOMICS}

Metabolomics techniques are recently becoming popular for biomarker discovery, also in the field of rheumatoid arthritis. Seven studies were found in PubMed in which metabolomics platforms were used to find metabolite profiles that distinguish rheumatoid arthritis patients from healthy controls. Four of these papers are dealing with synovial fluid samples (Williamson et al., 1989; Naughton et al., 1993a; Naughton et al., 1993b; Meshitsuka et al., 1999), two papers with human serum samples (Lauridsen et al., 2010; Wietmarschen et al., 2009) and one paper with mouse serum samples (Weljie et al., 2007).

The synovial fluid of healthy people show elevated lactate levels, indicating a hypoxic status in the joint. In RA patients this level of lactate is more elevated, glucose levels are diminished and ketone bodies are detectable indicating an even more hypoxic state and the utilization of fatty acids for energy. The inflamed joint of RA patients also contained lipoprotein associated fatty acids such as VLDL triacylglycerols, LDL, HDL, cholesterol and HDL phospholipids, which are not able to enter the joint space in healthy conditions (Naughton et al., 1993a).

In serum of $\mathrm{K} / \mathrm{BxN}$ arthritis mice several metabolic changes have been found compared to healthy control mice using NMR spectroscopy. Some lipid, fatty acid and carbohydrate metabolism markers were found: Glycerol, Choline, 2-hydroxybutyrate, acetylcarnitine, TMAO. Energy metabolism markers 2-oxoglutarate, acetylcarnitine were detected. The markers xanthine, hypoxanthine, uridine, uracil, TMOA are related to nucleic acid metabolism. Glutamate, serine, phenylalanine, glycine, methionine, asparagine are related to amino acid metabolism. Methionine, glycine, taurine, xanthine, hypoxanthine, TMAO, acetylcarnitine are important for dealing with oxidative stress (Weljie et al., 2007).

Plasma from human RA patients has been shown to contain elevated lactate and cholesterol levels and decreased HDL levels. Elevated plasma lactate levels have been associated with increased oxidative damage and lowered synovial $\mathrm{pH}$. Lower levels of HDL and consequently higher levels of cholesterol support the increased risk of cardiovascular diseases in RA patients (Lauridsen et al., 2010). The only study in which gas chromatography mass spectrometry (GC-MS) was used to detect metabolic differences between RA patients and healthy controls reported higher levels of heptanoic acid, I-Alanine, 2-oxy-butanoic acid, I-asparagine, palmitic acid and lower levels of 2-butenoic acid, 
undecanoic acid, d-glucuronic acid, ribitol, stearic acid in RA patients (van Wietmarschen et al., 2009).

\section{CONNECTING THE DOTS}

In the following section we will discuss the literature findings concerning metabolites and RA in an attempt to uncover relationships between metabolites and biological mechanisms relevant for studying RA. The interpretation of metabolite information from various sources is challenging for several reasons. For instance, publications often describe the role of classes of metabolites instead of specifying individual metabolites with appropriate database identifiers. Pathway analysis tools are not able to handle groups or families of metabolites. A second challenge is that many measured metabolites are not yet present in the databases connected to pathways analysis software. Therefore the actual list of metabolites that can be analyzed by pathway construction tools usually represents only part of what is actually known.

For 20 of the 25 metabolites related to human plasma changes in RA an identifier could be found in the Kyoto Encyclopedia of Genes and Genomes (KEGG). These metabolites were imported in the Ingenuity Pathway Analysis software and used for the subsequent analysis (Gehlenborg et al., 2010). Metabolites with higher levels in RA patients were given a fold change value of 2 while the metabolites with lower levels in RA were given a fold change value of minus 2 because the actual values could not be determined and compared between studies based on the literature.

Several of the compounds were found to be involved with the key molecular and cellular functions cellular development, cellular growth and proliferation, cell death, lipid metabolism and molecular transport. In addition, the set of compounds was found to be mostly related to metabolic disease, inflammatory response, cardiovascular disease, endocrine system disorders and gastrointestinal disease.

The network (Fig. 1) most closely related to the data set was characterized by the top functions cell death, lipid metabolism and small molecule biochemistry containing 9 of the reviewed metabolites: arachidonic acid, ascorbic acid, beta-carotene, cholecalciferol, hydrocortisone, keratan sulfate, melatonin, palmitic acid and stearic acid. These nine metabolites are highly connected to a number of canonical pathways related to immune functions, the production of nitric oxygen and reactive oxygen species in macrophages and pathways involved in arthritis. Specifically the role of macrophages, fibroblasts, endothelial cells, osteoblasts, osteoclasts and chondrocytes in rheumatoid arthritis are connected to this network as well as the role of IL-17 in arthritis and IL-12 signaling and production in macrophages. 


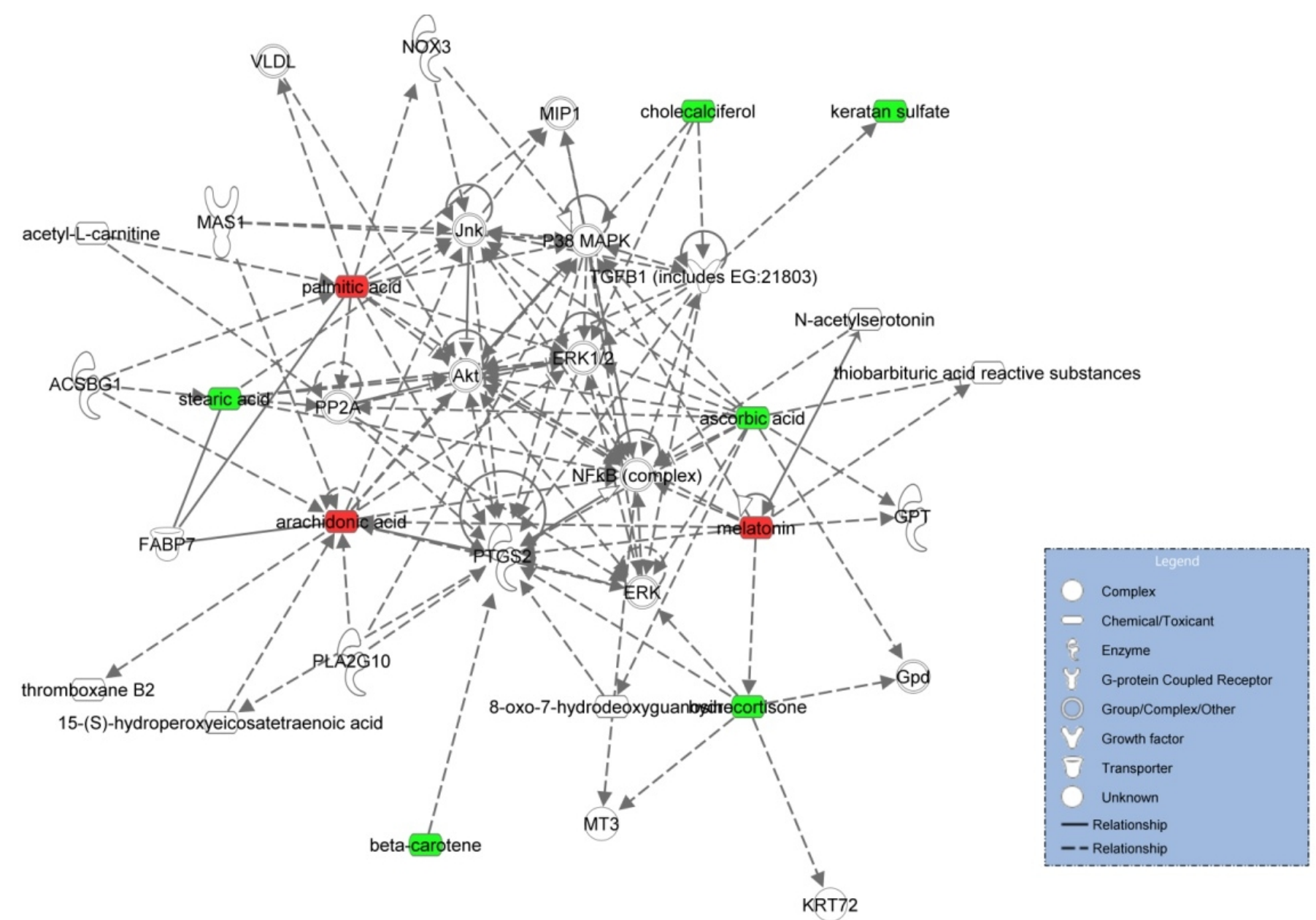

Fig. 1. Ingenuity pathway analysis of the metabolites discussed in this review that are related to rheumatoid arthritis Relationships are based on literature mining. Red colored nodes indicate up-regulated metabolites and green colored nodes indicate downregulated metabolites 


\section{FINAL REMARKS}

Many metabolites have been reported to play a role in rheumatoid arthritis. This review indicates groups of metabolites that could be interesting for metabolomics studies into the mechanism of and possible treatment options for rheumatoid arthritis. Eicosanoids, fatty acids, lipids, trace elements, vitamins and several hormones are interesting candidates for elucidating the mechanism of RA. Pathway analysis has provided an indication of biological processes related to the discussed collection of RA related metabolites. This review suggests that more clinical studies are needed to elucidate the effects of vitamin supplementation on RA activity and progression. In addition, circadian rhythms in hormone production and other metabolite levels are important to consider. For instance, the timing of glucocorticoid treatment is essential for obtaining an optimal effect (Cutolo, 2008). This review also shows the complexity of the immune and hormonal processes that are changed in rheumatoid arthritis patients, suggesting that a move from single-target therapy towards multi-target therapy is needed. Finally, a more personalized medicine approach is needed to address the differences in the expression, severity, progression and metabolic changes of RA for each individual.

\section{COMPETING INTERESTS}

Authors have declared that no competing interests exist.

\section{REFERENCES}

Aaseth, J. (1998). Rheumatoid arthritis and metal compounds - perspectives on the role of oxygen radical detoxification. The Analyst., 123.

ACR. (2002). Guidelines for the management of rheumatoid arthritis: 2002 Update. Arthritis Rheum., 46(2), 328-46.

Adam, O., Beringer, C., Kless, T., et al. (2003). Anti-inflammatory effects of a low arachidonic acid diet and fish oil in patients with rheumatoid arthritis. Rheumatol Int., 23(1), 27-36.

Aman, S., Risteli, J., Luukkainen, R., et al. (1999). The value of synovial fluid analysis in the assessment of knee joint destruction in arthritis in a three year follow up study. Ann Rheum Dis., 58(9), 559-62.

Arnson, Y., Amital, H., Shoenfeld, Y. (2007). Vitamin D and autoimmunity: new aetiological and therapeutic considerations. Ann Rheum Dis., 66(9), 1137-42.

Breslau, N.A., Brinkley, L., Hill, K.D., Pak, C.Y. (1988). Relationship of animal protein-rich diet to kidney stone formation and calcium metabolism. J Clin Endocrinol Metab., 66(1), 140-6.

Burger, D., Dayer, J. (2002). High-density lipoprotein-associated apolipoprotein A-I: the missing link between infection and chronic inflammation? Autoimmun Rev., 1(1-2), 111-7.

Capellino, S., Straub, R.H. (2008). Neuroendocrine immune pathways in chronic arthritis. Best Pract Res Clin Rheumatol., 22(2), 285-97.

Cesur, M., Ozbalkan, Z., Temel, M.A., Karaarslan, Y. (2007). Ethnicity may be a reason for lipid changes and high Lp(a) levels in rheumatoid arthritis. Clin Rheumatol., 26(3), 355-61. 
Chan, J.M., Giovannucci, E., Andersson, S.O., et al. (1998). Dairy products, calcium, phosphorous, vitamin $\mathrm{D}$ and risk of prostate cancer (Sweden). Cancer Causes Control., 9(6), 559-66.

Charni, N., Juillet, F., Garnero, P. (2005). Urinary type II collagen helical peptide (HELIX-II) as a new biochemical marker of cartilage degradation in patients with osteoarthritis and rheumatoid arthritis. Arthritis Rheum., 52(4),1081-90.

Christakos, S., Dhawan, P., Liu, Y., Peng, X., Porta, A. (2003). New insights into the mechanisms of vitamin D action. J Cell Biochem., 88(4), 695-705.

Christgau, S., Garnero, P., Fledelius, C., et al. (2001). Collagen type II C-telopeptide fragments as an index of cartilage degradation. Bone., 29(3), 209-15.

Cleland, L.G., James, M.J. (1997). Rheumatoid arthritis and the balance of dietary N-6 and N-3 essential fatty acids. Br J Rheumatol., 36(5), 513-4.

Cline, M.S., Smoot, M., Cerami, E., et al. (2007). Integration of biological networks and gene expression data using Cytoscape. Nat Protoc., 2(10), 2366-82.

Comstock, G.W., Burke, A.E., Hoffman, S.C., et al. (1997). Serum concentrations of alpha tocopherol, beta carotene, and retinol preceding the diagnosis of rheumatoid arthritis and systemic lupus erythematosus. Ann Rheum Dis., 56(5), 323-5.

Cutolo, M., Otsa, K., Laas, K., et al. (2006). Circannual vitamin d serum levels and disease activity in rheumatoid arthritis: Northern versus Southern Europe. Clin Exp Rheumatol., 24(6), 702-4.

Cutolo, M., Otsa, K., Uprus, M., Paolino, S., Seriolo, B. (2007). Vitamin D in rheumatoid arthritis. Autoimmun Rev., 7(1), 59-64.

Cutolo, M., Straub, R.H. (2008). Circadian rhythms in arthritis: hormonal effects on the immune/inflammatory reaction. Autoimmunity Reviews, 7(3), 223-228.

Cutolo, M., Plebani, M., Shoenfeld, Y., Adorini, L., Tincani, A. (2011). Vitamin D endocrine system and the immune response in rheumatic diseases. Vitam. Horm., 86, 327-351.

Cutolo, M., Straub, R.H. (2008). Circadian rhythms in arthritis: hormonal effects on the immune/inflammatory reaction. Autoimmun Rev., 7(3), 223-8.

Cutolo, M., Villaggio, B., Otsa, K., et al. (2005). Altered circadian rhythms in rheumatoid arthritis patients play a role in the disease's symptoms. Autoimmun Rev., 4(8), 497502.

Devchand, P.R., Keller, H., Peters, J.M., et al. (1996). The PPARalpha-leukotriene B4 pathway to inflammation control. Nature, 384(6604), 39-43.

Dursunoğlu, D., Evrengül, H., Polat, B., et al. (2005). Lp(a) lipoprotein and lipids in patients with rheumatoid arthritis: serum levels and relationship to inflammation. Rheumatol Int., 25(4), 241-5.

Forestier, J. (1949). Copper and gold salts in rheumatoid arthritis. Ann Rheum Dis., 8(2), $132-4$

Funk, C.D. (2001). Prostaglandins and leukotrienes: advances in eicosanoid biology. Science., 294(5548), 1871-5.

Gehlenborg, N., O'Donoghue, S.I., Baliga, N.S., Goesmann, A., Hibbs, M.A., Kitano, H., Kohlbacher, O., et al. (2010). Visualization of omics data for systems biology. Nature Methods, 7(3 Suppl), S56-68.

Glocker, M.O., Guthke, R., Kekow, J., Thiesen, H. (2006). Rheumatoid arthritis, a complex multifactorial disease: on the way toward individualized medicine. Med Res Rev., 26(1), 63-87.

Hakala, M., Aman, S., Luukkainen, R., et al. (1995). Application of markers of collagen metabolism in serum and synovial fluid for assessment of disease process in patients with rheumatoid arthritis. Ann Rheum Dis., 54(11), 886-90.

Halsted, J.A., Smith, J.C. (1970). Plasma-zinc in health and disease. Lancet, 1(7642), 322-4. 
Heliövaara, M., Knekt, P., Aho, K., et al. (1994). Serum antioxidants and risk of rheumatoid arthritis. Ann Rheum Dis., 53(1), 51-3.

Hochberg, M.C., Silman, A.J., Smolen, J.S., Weinblatt, M.E., Weisman, M.H. (2007). Rheumatology, 2-Volume Set: with Image Library Bind-In CD-ROM. 4th ed. Mosby, 2288.

Hoffmann, P.R., Berry, M.J. (2008). The influence of selenium on immune responses. Mol Nutr Food Res., 52(11), 1273-80.

Honkanen, V., Lambergallardt, C., Vesterinen, M., et al. (1991). Plasma zinc and copper concentrations in rheumatoid-arthritis- influence of dietary factors and disease-activity. American Journal of Clinical Nutrition., 54(6), 1082-1086.

Hönscheid, A., Rink, L., Haase, H. (2009). T-lymphocytes: a target for stimulatory and inhibitory effects of zinc ions. Endocr Metab Immune Disord Drug Targets, 9(2), 132144.

Hulthe, J., Fagerberg, B. (2002). Circulating oxidized LDL is associated with subclinical atherosclerosis development and inflammatory cytokines (AIR Study). Arterioscler Thromb Vasc Biol., 22(7), 1162-7.

Huynh, H., Pollak, M., Zhang, J.C. (1998). Regulation of insulin-like growth factor (IGF) II and IGF binding protein 3 autocrine loop in human PC-3 prostate cancer cells by vitamin D metabolite 1,25(OH)2D3 and its analog EB1089. Int J Oncol., 13(1), 137-43.

Hyka, N., Dayer, J.M., Modoux, C., et al. (2001). Apolipoprotein A-I inhibits the production of interleukin-1beta and tumor necrosis factor-alpha by blocking contact-mediated activation of monocytes by T lymphocytes. Blood., 97(8), 2381-9.

Kalpakcioglu, B., Senel, K. (2008). The interrelation of glutathione reductase, catalase, glutathione peroxidase, superoxide dismutase and glucose-6-phosphate in the pathogenesis of rheumatoid arthritis. Clin Rheumatol., 27(2), 141-5.

Kersten, S., Desvergne, B., Wahli, W. (2000). Roles of PPARs in health and disease. Nature, 405(6785), 421-4.

Klickstein, L.B., Shapleigh, C., Goetzl, E.J. (1980). Lipoxygenation of arachidonic acid as a source of polymorphonuclear leukocyte chemotactic factors in synovial fluid and tissue in rheumatoid arthritis and spondyloarthritis. J Clin Invest., 66(5), 1166-70.

Kojo, H., Fukagawa, M., Tajima, K., et al. (2003). Evaluation of human peroxisome proliferator-activated receptor (PPAR) subtype selectivity of a variety of antiinflammatory drugs based on a novel assay for PPAR delta(beta). J Pharmacol Sci., 93(3), 347-55.

Kremer, J.M., Lawrence, D.A., Jubiz, W., et al. (1990). Dietary fish oil and olive oil supplementation in patients with rheumatoid arthritis. Clinical and immunologic effects. Arthritis Rheum., 33(6), 810-20.

Kritchevsky, D., Tepper, S.A., Czarnecki, S.K., Klurfeld, D.M. (1982). Atherogenicity of animal and vegetable protein. Influence of the lysine to arginine ratio. Atherosclerosis, 41(2-3), 429-31.

Labrecque, G., Bureau, J.P., Reinberg, A.E. (1995). Biological rhythms in the inflammatory response and in the effects of non-steroidal anti-inflammatory drugs. Pharmacology \& Therapeutics, 66(2), 285-300.

Lakatos, J., Hárságyi, A. (1988). Serum total, HDL, LDL cholesterol, and triglyceride levels in patients with rheumatoid arthritis. Clin Biochem., 21(2), 93-6.

Langman, C.B. (1989). Calcitriol metabolism during chronic metabolic acidosis. Semin Nephrol., 9(1), 65-71.

Lauridsen, M.B., Bliddal, H., Christensen, R., et al. (2010). 1H NMR spectroscopy-based interventional metabolic phenotyping: a cohort study of rheumatoid arthritis patients. J. Proteome Res., 9(9), 4545-4553. 
Lazarevic, M.B., Vitic, J., Myones, B.L., et al. (1993). Antilipoprotein antibodies in rheumatoid arthritis. Semin Arthritis Rheum., 22(6), 385-91.

Lewis, A.J. (1984). The role of copper in inflammatory disorders. Agents Actions, 15(5-6), 513-9.

Lipsky, P.E., Ziff, M. (1977). Inhibition of antigen- and mitogen-induced human lymphocyte proliferation by gold compounds. J Clin Invest., 59(3), 455-66.

Mattingly, P.C., Mowat, A.G. (1982). Zinc sulphate in rheumatoid arthritis. Ann Rheum Dis., 41(5), 456-7.

Merlino, L.A., Curtis, J., Mikuls, T.R., et al. (2004). Vitamin D intake is inversely associated with rheumatoid arthritis: results from the lowa women's health study. Arthritis Rheum., 50(1), 72-77.

Meshitsuka, S., Yamazaki, E., Inoue, M., et al. (1999). Nuclear magnetic resonance studies of synovial fluids from patients with rheumatoid arthritis and osteoarthritis. Clin. Chim. Acta., 281(1-2), 163-167.

Naughton, D., Whelan, M., Smith, E.C., et al. (1993a). An investigation of the abnormal metabolic status of synovial fluid from patients with rheumatoid arthritis by high field proton nuclear magnetic resonance spectroscopy. FEBS Lett., 317(1-2), 135-8.

Naughton, D.P., Haywood, R., Blake, D.R., et al. (1993b). A comparative evaluation of the metabolic profiles of normal and inflammatory knee-joint synovial fluids by high resolution proton NMR spectroscopy. FEBS Lett., 332(3), 221-5.

Neeck, G., Federlin, K., Graef, V., Rusch, D., Schmidt, K.L. (1990). Adrenal secretion of cortisol in patients with rheumatoid arthritis. J Rheumatol., 17(1), 24-9.

O'Connor, J.C., McCusker, R.H., Strle, K., et al. (2008). Regulation of IGF-I function by proinflammatory cytokines: at the interface of immunology and endocrinology. Cell Immunol., 252(1-2), 91-110.

Oelzner, P., Müller, A., Deschner, F., et al. (1998). Relationship between disease activity and serum levels of vitamin D metabolites and PTH in rheumatoid arthritis. Calcif. Tissue Int., 62(3), 193-198.

Pattison, D.J., Silman, A.J., Goodson, N.J., et al. (2004). Vitamin C and the risk of developing inflammatory polyarthritis: prospective nested case-control study. Ann Rheum Dis., 63(7), 843-7.

Poole, A.R., lonescu, M., Swan, A., Dieppe, P.A. (1994). Changes in cartilage metabolism in arthritis are reflected by altered serum and synovial fluid levels of the cartilage proteoglycan aggrecan. Implications for pathogenesis. J Clin Invest., 94(1), 25-33.

Rasker, J.J., Kardaun, S.H. (1982). Lack of beneficial effect of zinc sulphate in rheumatoid arthritis. Scand J Rheumatol., 11(3), 168-70.

Rister, M., Bauermeister, K., Gravert, U., Gladtke, E. (1978). Superoxide-dismutase deficiency in rheumatoid arthritis. Lancet., 1(8073), 1094.

Roubenoff, R., Roubenoff, R.A., Selhub, J., et al. (1995). Abnormal vitamin B6 status in rheumatoid cachexia. Association with spontaneous tumor necrosis factor alpha production and markers of inflammation. Arthritis Rheum., 38(1), 105-9.

Sakuma, T., Miyamoto, T., Jiang, W., et al. (2003). Inhibition of peroxisome proliferatoractivated receptor alpha signaling by vitamin $\mathrm{D}$ receptor. Biochem Biophys Res Commun., 312(2), 513-9.

Smolen, J.S., Aletaha, D.. (2005). Challenges of predicting treatment response in patients with rheumatoid arthritis. Nature Clinical Practice. Rheumatology, 1(2), 62-63.

Tarp, U., Overvad, K., Thorling, E.B., Graudal, H., Hansen, J.C. (1985). Selenium treatment in rheumatoid arthritis. Scand J Rheumatol., 14(4), 364-8.

Tudor, R., Zalewski, P.D., Ratnaike, R.N. (2005). Zinc in health and chronic disease. J Nutr Health Aging., 9(1), 45-51. 
van der Greef, J., Martin, S., Juhasz, P., et al. (2007). The art and practice of systems biology in medicine: mapping patterns of relationships. J Proteome Res., 6(4), 154059.

van Halm, V.P., Nielen, M.M.J., Nurmohamed, M.T., et al. (2007). Lipids and inflammation: serial measurements of the lipid profile of blood donors who later developed rheumatoid arthritis. Ann Rheum Dis., 66(2), 184-8.

van Wietmarschen, H., Yuan, K., Lu, C., et al. (2009). Systems biology guided by Chinese medicine reveals new markers for sub-typing rheumatoid arthritis patients. J Clin Rheumatol., 15(7), 330-337.

Weljie, A.M., Dowlatabadi, R., Miller, B.J., Vogel, H.J., Jirik, F.R. (2007). An inflammatory arthritis-associated metabolite biomarker pattern revealed by $1 \mathrm{H}$ NMR spectroscopy. J. Proteome Res., 6(9), 3456-3464.

Welsing, P.M.J., Fransen, J., van Riel, P.L.C.M. (2005). Is the disease course of rheumatoid arthritis becoming milder? Time trends since 1985 in an inception cohort of early rheumatoid arthritis. Arthritis Rheum., 52(9), 2616-24.

Williamson, M.P., Humm, G., Crisp, A.J. (1989). 1H nuclear magnetic resonance investigation of synovial fluid components in osteoarthritis, rheumatoid arthritis and traumatic effusions. Br J Rheumatol., 28(1), 23-7.

Wishart, D.S., Tzur, D., Knox, C., et al. (2007). HMDB: the human metabolome database. Nucleic Acids Res., 35(Database issue): D521-6.

(C) 2012 Wietmarschen and Greef; This is an Open Access article distributed under the terms of the Creative Commons Attribution License (http://creativecommons.org/licenses/by/3.0), which permits unrestricted use, distribution, and reproduction in any medium, provided the original work is properly cited. 